reader without a modern scientific training; as an account of the science of our century it clearly does not give any kind of coverage, but it does indicate seminal points. Joliot on atomic weapons has contemporary resonance, but it is perhaps surprising that there is no sequel to the naturalists or to Mendel, given the importance for us of ecology and genetics.

We thus have a book full of stimulating insights, something to be recommended to students or to the general reader who has some idea of what science is and was about. We find some memorable episodes such as the mediaeval attempt to make theology a science, as described by Paul Benôit; but probably for scientists, the emphasis on beginnings and times remote from our own will make the book particularly desirable. These lively accounts and the anonymous translator is to be congratulated - take the reader a long way from the brief run-throughs of recent history to be found in science textbooks or encyclopaedias. And the book also gives professionals in history and philosophy of science something to engage with; it is very welcome.

David Knight is in the Department of Philosophy, University of Durham, 50 Old Elvet, Durham DH1 3HN, UK.

\title{
The last big bang?
}

Mike A'Hearn

\begin{abstract}
Rogue Asteroids and Doomsday Comets: The Search for the Million Megaton Menace that Threatens Life on Earth. By Duncan Steel. Wiley: 1995. Pp. 308. \$24.95, £16.99.
\end{abstract}

WiLl we or our descendants all die when an extraterrestrial object comes hurtling down onto Earth? This question was brought to the fore by the enormous publicity surrounding the collision of the fragments of comet Shoemaker-Levy 9 with Jupiter in July 1994. Even before these events, the question had stimulated some astronomers to propose searches to identify all large asteroids that might hit Earth in the foreseeable future. The question had also stimulated developers of nuclear weapons to propose mechanisms for deflecting such asteroids before they collide with Earth. At the behest of the US Congress, NASA has appointed several committees to consider detection and deflection of potentially hazardous asteroids, the most recent of which, the Shoemaker Committee, has just released its report. Meanwhile, the International Astronomical Union has also appointed a working group to consider what actions are scientifically appropriate in studying and detecting
near-Earth objects.

As someone engaged on the periphery of this issue, it seems clear to me that certain aspects of the problem have been investigated thoroughly and are well understood, whereas others have been almost ignored and so are not understood. It is technically possible to carry out a search, on a timescale of ten years or so, to detect 90 per cent of the near-Earth asteroids with radii greater than $1 \mathrm{~km}$ that might hit Earth in the foreseeable future.

On the other hand, the hazard due to long-period comets is poorly understood and we do not know how to detect these more than a year or two in advance. Similarly, we do not fully understand the nature of the hazard even from asteroids of a given size. Studies of last year's Jupiter impacts (discussed in a lengthy epilogue to the book) show the importance of a process previously not even considered: when material ejected during the impact falls back down onto the atmosphere, it dramatically heats the atmosphere over very large areas.

It is also unclear how the public views the risk of an extraterrestrial impact compared with that of, say, an acroplane crash. Statistically, the risks of death from the two causes would seem to be of the same order of magnitude. Extraterrestrial impacts are, of course, much less frequent but have a dramatically greater effect, leading to a huge uncertainty in the actual number of deaths as well as a notable difference in public perception of the risk.

Steel aims to heighten public awareness of the risk and thus develop political support for funding the searches and other studies that might ultimately allow us to save ourselves or our descendants from a worldwide catastrophe. He writes about facts that are accepted by most astronomers and ideas that are wildly speculative, as well as about everything in between. There are vast uncertainties in many of the predictions and he points out many areas where astronomers are in disagreement. Examples of past impacts include the generally understood small impacts, such as Meteor Crater in Arizona and the explosion above Tunguska in 1908 , but Steel also writes about other impacts, the effects of which are not so widely accepted. It seems to me that most astronomers accept the theory that the impact that created the Chicxulub crater in the Yucatan also caused the extinction of the dinosaurs at the end of the Cretaceous period, although this is apparently less widely accepted among palaeontologists, some of whom still argue for a more gradual extinction. Unclear to both astronomers and palaeontologists is whether large craters and extinctions are really periodic.

With its wide range of speculation, every scientist, whether astronomer or geologist, palaeontologist or archaeologist, will disagree with one or another of the suggestions in this engaging and worthwhile book. Steel's proposal that the Taurid meteor showers sparked off the building of the first stage of Stonehenge will certainly charge up many readers, some positively and others negatively. This is, of course, his purpose in writing the book - to encourage others to think about the problem. The basic question is whether we should be terrified at the prospect of a large impact. Should we spend the money now to begin the search for potential impact objects and find out more about this particular hazard - a hazard that is undoubtedly real but of uncertain magnitude?

Mike A'Hearn is at the Astronomy Program, University of Maryland, Stadium Drive, College Park, Maryland 20742, USA. 